Bio - grafia. Escritos sobre la Biología y su Enseñanza. ISSN 2027

Edición Extraordinaria. p.p. 970- 981

Memorias del VIII Encuentro Nacional de Experiencias en Enseñanza de la Biología y la Educación Ambiental. III Congreso Nacional de Investigación en Enseñanza de la Biología.

\title{
La formación profesional de los profesores de secundaria que enseñan Biología en México: el caso del Distrito Federal
}

\author{
Martínez Hernández, María de la Luz ${ }^{1}$ y Rodríguez Pineda, Diana Patricia ${ }^{2}$
}

\section{Resumen.}

Con el propósito de caracterizar a los docentes que imparten Biología en la Educación Secundaria en México se hace una investigación documental, en la que se empleó un método comparativo. Los criterios de búsqueda -en el ámbito de la secundaria-que guiaron el trabajo fueron: formación profesional, formación docente, profesores de ciencias naturales y de biología. Los hallazgos se organizaron en dos ámbitos: contexto internacional -particularmente Europa y Latinoamérica- y, contexto nacional -México y en particular el Distrito Federal-. El segundo ámbito, es decir el caso de México, se organizó en tres niveles: en el primero presentamos la distribución de los docentes que cuentan con formación inicial como profesores -obtenida en las normales-, denominada formación simultánea $y$, la distribución de quienes tienen una formación inicial diferente -no normalistas-, conocida como formación consecutiva; en el segundo se abordó la frecuencia de perfiles profesionales de los profesores de ciencias naturales y; en el tercero se ubicó sólo la distribución de perfiles de docentes de Biología. Tanto en el contexto internacional, como en el nacional, los hallazgos indican que la tendencia es incorporar profesionistas libres al magisterio, favoreciendo una formación consecutiva. Los perfiles dominantes de los docentes de Biología de secundaria en México, coinciden en los distintos reportes de investigación, siendo principalmente biólogos, licenciados en ciencias naturales, médicos y odontólogos. Tal diversidad parece tener un origen social y consecuencias pedagógicas, como el bajo nivel académico de los alumnos.

Palabras clave: Formación docente, educación secundaria, enseñanza de la Biología, perfiles profesionales

Abstract. In order to characterize the biology teachers who work in the secondary education in Mexico, a documentary research as a comparative method was used, the 
Bio - grafia. Escritos sobre la Biología y su Enseñanza. ISSN 2027

Edición Extraordinaria. p.p. 970- 981

Memorias del VIII Encuentro Nacional de Experiencias en Enseñanza de la Biología y la Educación Ambiental. III Congreso Nacional de Investigación en Enseñanza de la Biología.

searching criteria that guided the work were: professional training, teaching training, natural sciences and biology teachers, findings were organized into two areas and three levels. Findings where separate into two contexts, international context, particularly Europe and Latin America, and national context, Mexico and specially the Mexico City. It was organized into three levels, in the first we present the distribution of teachers with a starting formation in education (obtained in a school of education.), called simultaneous formation and, the distribution of people which have a different starting formation (not members of said school), known as a consecutive formation. In the second level the frequency of professional profiles of the science teachers are addressed. And in the third level only the Biology teachers' profile distribution was used. It was found that the tendency in the world and in Mexico is to incorporate teachers without an education degree, being professional with consecutive training. The dominant profiles of secondary school biology teachers of México, according to various reports, are biologists, graduates in natural sciences, medics and dentists. The causes of this diversity have a social origin, and an educational consequence, causing a low educational level in students.

Keywords: Teacher training, secondary education, teaching biology, professional profiles

1 SEP-CSES/UPN Doctorado en Educación, Línea Educación en Ciencias. fluzma@hotmail.com

${ }^{2}$ Universidad Pedagógica Nacional dpineda@upn.mx

Introducción

Antes de abordar el asunto de la formación profesional de los profesores de secundaria que enseñan Biología en México, conviene hacer una breve mención al origen de la escuela secundaria en México, dado que este es el ámbito de nuestro objeto de estudio. La educación secundaria en México nace en 1925, gracias a la propuesta de Moisés Sáenz de la Garza, quien propuso la creación de un nivel intermedio entre la Educación Elemental Superior y la Escuela Nacional Preparatoria. La finalidad de ésta, era preparar de manera temprana a los alumnos para el trabajo haciendo énfasis en la formación técnica. En la década de los 30 s, durante la educación socialista se refirma su sentido técnico y de formación para el trabajo incorporando al sistema las escuelas técnicas y agropecuarias y en las ciudades las escuelas pre-vocacionales, en 1959 el Sistema Educativo Nacional se expande con el plan de 11 años que promueve la universalidad de la educación elemental 
Bio - grafia. Escritos sobre la Biología y su Enseñanza. ISSN 2027

Edición Extraordinaria. p.p. 970- 981

Memorias del VIII Encuentro Nacional de Experiencias en Enseñanza de la Biología y la Educación Ambiental. III Congreso Nacional de Investigación en Enseñanza de la Biología.

buscando dar educación primaria a todos los demandantes de México, eso presionó al sistema de secundarias que se expande ante la demanda (Martínez, 1997).

Tendencias de formación de los docentes de escuela secundaria.

A nivel mundial, los modelos de formación docente; simultáneos y consecutivos (Esteve, 2006), Los modelos de formación simultánea son aquellos en las que el futuro profesor estudia los contenidos científicos y recibe la formación científica necesaria para comunicarlos en el aula y profesional más adecuada al mismo tiempo. Los modelos de formación consecutivo, primero dotan al profesor de una formación académica sobre los contenidos científicos que se van a transmitir, y después se les da una formación profesional sobre los conocimientos pedagógicos y psicológicos que necesitan para enseñar ese contenido en las aulas, éstos modelos han convivido hasta la actualidad. Los encontramos tanto en países del primer mundo con altos niveles de escolaridad, como en países en vías de desarrollo con recursos limitados y niveles de escolaridad baja en su población. En Europa se reporta la inserción de docentes con modelo consecutivo en Gran Bretaña, Alemania y España, sólo por señalar algunos casos.

Para el caso de la enseñanza de la ciencia, Gran Bretaña, tiene dificultades para cubrir la demanda de docentes especializados en materias tecno científicas, los egresados de institutos y universidades encuentran trabajo en su ámbito de desarrollo profesional. En España, se reportó un incremento de la oferta de maestros habilitados a partir de la LOGSE, -en materias tecno-científicas-, debido al incremento del desempleo (Esteve, 2006, p.45).

En América Latina, el modelo de formación simultánea y consecutiva es constante en Argentina, Brasil, Colombia y Chile. En todos ellos la tendencia es a dejar los modelos de formación docente en escuelas "tradicionales", lo que llamaríamos normales, pero a su vez recurren a profesionistas libres para prepararlos como docentes de materias tecno científicas en el nivel de secundaria, lo han institucionalizado para paliar el desempleo de sus universitarios (OEI, 2003; Murillo, 2006). 
Bio - grafia. Escritos sobre la Biología y su Enseñanza. ISSN 2027

Edición Extraordinaria. p.p. 970- 981

Memorias del VIII Encuentro Nacional de Experiencias en Enseñanza de la Biología y la Educación Ambiental. III Congreso Nacional de Investigación en Enseñanza de la Biología.

En México, la formación de profesores para enseñar Biología a principios de siglo XX se dio en la Escuela Nacional de Altos Estudios de la Universidad Nacional Autónoma de México (UNAM), a su desaparición se crea la Escuela Normal Superior (ENS) cuna de la identidad del magisterio de didáctica especializada. Sin embargo, el número de escuelas secundarias superaba la oferta de egresados de la ENS, por lo que desde su formación recurrió a profesionistas libres, término que da Arnaut (1998) a los profesionales de diversos ámbitos que no recibe una instrucción para servir al Estado -por ejemplo militares y maestros-.

Las problemáticas sociales que han promovido que los docentes de escuela secundaria en México, tengan perfiles diversos y formación para la docencia consecutiva, se han incrementado; desempleo, falta de desarrollo del aparato industrial, dependencia tecnológica, empobrecimiento de la figura del docente. A ello se ha sumado la baja en las matrículas de las escuelas formadoras de maestros especializados para secundaria. La ENS reporta sólo dos grupos por generación, número de estudiantes que no alcanza a cubrir la demanda de estos especialistas en secundarias, al mismo tiempo se ha incrementado la población que egresa de las Instituciones de Educación Superior (IES) no formadoras de docentes. La alta población de docentes sin formación pedagógica de origen (modelo simultáneo), está relacionado con el bajo nivel de logro académico de acuerdo a los resultados de PISA, de la escuela secundaria en áreas como las ciencias naturales y Biología (INEE, 2010). Por ello se hace necesario conocer:

¿Cuáles son los perfiles profesionales de los docentes que imparten Biología en escuela secundaria?

La investigación tiene como objetivo documentar la diversidad de la planta docente que imparte biología, aportar un diagnóstico de los maestros que la imparten.

\section{Estrategia Metodológica}

Se hizo una revisión documental, tomando como criterio tres categorías de análisis: formación profesional, formación docente del maestro de secundaria $y$, profesores de ciencias naturales -particularmente de Biología- de ese nivel. Se empleó un método comparativo, que implicó contrastar de forma sistemática la información encontrada. La 
Bio - grafia. Escritos sobre la Biología y su Enseñanza. ISSN 2027

Edición Extraordinaria. p.p. 970- 981

Memorias del VIII Encuentro Nacional de Experiencias en Enseñanza de la Biología y la Educación Ambiental. III Congreso Nacional de Investigación en Enseñanza de la Biología.

información se validó por el uso de su metodología explicita y/o bien por la cita de sus fuentes. Se recurrió a bibliotecas especializadas de universidades, bases de datos de revistas indexadas, y memorias arbitradas de congresos con comités científicos. Lo recabado se vació en una base de datos elaborada en Excel, con las categorías ya definidas como elemento de supra orden, número de documento, cita de la fuente en sistema APA, resumen no menor a 300 palabras, país y año. Con la información ordenada, se agrupó la información en dos ámbitos y tres niveles de inclusión, se generaron tablas concentradoras para describir el comportamiento de los datos y se recurrió a la base conceptual, para generar tanto explicaciones como argumentos de tipo analítico.

\section{Resultados}

\section{- Ámbito internacional -Europa y Latinoamérica-.}

Respecto a la formación profesional, se encontró que hay una tradición en la comunidad europea de ver a la universidad como nivel superior de educación, ligándola con las necesidades sociales y aparato productivo. La formación de docentes, se cuenta con modelos variados, lo mismo se tiene escuelas especializadas (España) como formación no especializada que requiere de niveles de estudio cada vez más demandante para acceder a la docencia, según el nivel es la exigencia académica (Holanda). La existencia de modelos consecutivos (Holanda) como simultáneos (España), no excluye que en ambos países se recurra a maestros habilitados. Los profesores de secundaria se forman en los dos modelos, sin embargo no encontramos reportes específicos sobre la diversidad de su planta docente en asignaturas tecno-científicas como las ciencias naturales en secundaria (Esteves, 2006).

En Latinoamérica, la formación profesional se da en los IES, separando la formación de docentes de la profesionistas libres. La formación de docentes se da tanto en universidades como en escuelas tradicionales, entendidas como normales. Se emplean modelos simultáneos, los nuevos modelos de formación que están dejando de lado a las normales, sobre especializa los niveles como en el caso de Colombia, o bien dentro de una 
Bio - grafia. Escritos sobre la Biología y su Enseñanza. ISSN 2027

Edición Extraordinaria. p.p. $970-981$

Memorias del VIII Encuentro Nacional de Experiencias en Enseñanza de la Biología y la Educación Ambiental. III Congreso Nacional de Investigación en Enseñanza de la Biología.

universidad como tal, una facultad o escuela es la encargada de formar docentes (Argentina y Chile). Los profesores de secundaria para la enseñanza de la ciencia, se reporta oficialmente que se preparan en facultades o escuelas especializadas, sin embargo al profundizar en la información señalan que conviven los modelos simultáneos (preferentemente) y consecutivos en zonas desprotegidas, incluso en caso Chile se menciona como una alternativa de empleo a profesionistas en dichas éstas (OEI, 2003; Murillo, 2006).

\section{- Ámbito nacional.}

El caso de México se dividió para su estudio en tres niveles: primero, normalistas (formación simultánea) vs universitarios (formación consecutiva); segundo, docentes de ciencias naturales y; tercero, maestros de Biología, todo ello en secundaria.

Primer nivel: perfil general de los docentes de secundaria. En cuanto a su formación profesional, está claramente diferenciado que las normales son las escuelas especializadas en preparar a maestros (escuela tradicional) y las universidades forman profesionistas libres, incluso la Universidad Pedagógica Nacional (UPN) en México, no forma docentes de origen, les llama profesionales de la educación, no docentes. En cuanto a los profesores que ingresan a secundaria se forman tanto en Normales de forma específica y simultánea, como en IES no normales con modelos consecutivos, ya en servicio. La distribución de docentes de formación consecutiva y simultánea, según algunos reportes tanto nivel nacional como local, el D.F. (Martínez, 1997; Sandoval, 2001; Quiroz, 2002;, Tarango, 2012), favorece a los primeros, que son profesionistas egresados de IES no normales (ver Tabla 1). 
Bio - grafia. Escritos sobre la Biología y su Enseñanza. ISSN 2027

Edición Extraordinaria. p.p. 970- 981

Memorias del VIII Encuentro Nacional de Experiencias en Enseñanza de la Biología y la Educación Ambiental. III Congreso Nacional de Investigación en Enseñanza de la Biología.

Tabla 1. Institución de formación de profesores de secundaria
\begin{tabular}{|c|c|c|}
\hline $\begin{array}{c}\text { AUTORI FORMACION DE LOS } \\
\text { DOCENTES }\end{array}$ & $\begin{array}{c}\text { NORMAL SUPERIOR } \\
\%\end{array}$ & $\begin{array}{c}\text { UNIVERSITARIO } \\
\%\end{array}$ \\
\hline Martinez (1997) & 20 & 80 \\
\hline $\begin{array}{c}\text { Medina (1999), Sandoval (2001), } \\
\text { Quiroz (2002) }\end{array}$ & 30 & 70 \\
\hline \begin{tabular}{c} 
Jiménez y Macias (2002) \\
\hline $\begin{array}{c}\text { González, Martínezy Velázquez } \\
\text { (2006) }\end{array}$
\end{tabular} & 25 & 75 \\
\hline Tarange (2012) & 40 & 44 \\
\hline Martínez, Más y Paz (2014) & 29 & 60 \\
\hline INEE (2015) & 25.9 & 40.1 \\
\hline
\end{tabular}

Segundo nivel: docentes de ciencias naturales. En cuanto a su formación profesional, las normales forman especialista en la enseñanza de diferentes disciplinas a partir de 1999 (Física, Química, Biología), pero sus generaciones entre 1984 y 1998 estudiaron por áreas, sin especificidad disciplinar, con el título genérico de Ciencias naturales. Las IES (no normales) siempre han formado por disciplina (al menos desde el siglo XIX). En formación docente, la ENS es la encargada de formar profesores especializados, modelo simultáneo, pero la necesidad de las escuelas secundarias a las que ingresan a trabajar los obliga a cubrir especialidades diferentes a las suya. Por ejemplo un maestro egresado de la especialidad de Biología, podrá cubrir Química o Física, sólo por ser maestro de "ciencias", éstos docentes especializados reciben una formación consecutiva en su entorno laboral. Caso similar es el de los maestros no normalistas, quienes con profesiones diversas que cubran el perfil requerido en el profesiograma SEP (2000), pueden impartir ciencias naturales, así un Ingeniero sufre una doble situación, primero deja de lado el horizonte profesional en su campo y posteriormente imparte asignaturas ajenas a su formación. Por ejemplo, un ingeniero por ser de "ciencias", puede impartir Biología. Al respecto, Rodríguez (2007), Blancas (2010), Flores-Camacho, Gallegos-Cazares, García-Franco, Vega-Murgía y García-Rivera en (2007), reportan los dos aspectos señalados, la gran diversidad de perfiles de los profesores que enseñan ciencias naturales en secundaria y su falta de especificidad disciplinar (ver Tabla 2). 
Bio - grafia. Escritos sobre la Biología y su Enseñanza. ISSN 2027

Edición Extraordinaria. p.p. 970- 981

Memorias del VIII Encuentro Nacional de Experiencias en Enseñanza de la Biología y la Educación Ambiental. III Congreso Nacional de Investigación en Enseñanza de la Biología.

ITabla 2. Perfiles dominantes de profesores de Ciencias Naturales
\begin{tabular}{|l|c|}
\hline \multicolumn{1}{|c|}{ DISCIPLINA DE FORMACION } & FRECUENCIA \\
\hline Biología & 37 \\
\hline Ingeniería & 35 \\
\hline Química & 33 \\
\hline Ciencias naturales & 26 \\
\hline Física & 24 \\
\hline Medicina & 9 \\
\hline Cirujano dentista & 8 \\
\hline Veterinario & 4 \\
\hline Español & 3 \\
\hline Matemáticas & 3 \\
\hline No especifica & 3 \\
\hline Ciencias de la educación & 4 \\
\hline Administración & 1 \\
\hline Arquitectura, & 1 \\
\hline Ciencias sociales & 1 \\
\hline Contador publico & 1 \\
\hline Agrónomo & 1 \\
\hline Diseño industrial & 1 \\
\hline Docencia universitaria & 1 \\
\hline Geografía & 1 \\
\hline Psicología & 1 \\
\hline Técnico & 1 \\
\hline
\end{tabular}

Tercer nivel: docentes de Biología. Los datos reportan que quienes tienen como perfil la formación profesional como maestros especializados en la enseñanza de la biología, son formados en la ENS, en tanto que los docentes con otros perfiles, han estudiado en escuelas diversas, no todas IES, ya que algunos tienen estudios a nivel técnico.

El docente que enseña Biología en secundaria ha sido preparado en la ENS, que históricamente ha seguido el modelo simultáneo, la ENS ha formado: maestros de ciencias biológicas (1936), maestros de Biología (1941), licenciados en ciencias naturales (1984), licenciados en la enseñanza de la Biología (1999). Todos ellos han tenido elementos para enseñar su disciplina de forma específica, salvo el plan 1984, pero podían optar por especializarse, ya en servicio en Biología. Por otro lado la UNAM desde 1939 forma Biólogos en la Facultad de Ciencias; al igual que el Instituto Politécnico Nacional (IPN), en la Escuela Nacional de Ciencias Biológicas (ENCB) y; la Universidad Autónoma Metropolitana (UAM), en la división de ciencias biológicas, todos ellos especializados en investigación del origen, evolución, distribución y fenómenos biológicos. Pero éstos no son los únicos perfiles profesionales de maestros que enseñan biología en secundaria. Rico (2006) y Martínez, Más y Paz (2013), reportan amplia diversidad de carreras de formación inicial de los docentes, pero al mismo tiempo acusan alta dominancia, es decir, son pocos los 
Bio - grafia. Escritos sobre la Biología y su Enseñanza. ISSN 2027

Edición Extraordinaria. p.p. 970- 981

Memorias del VIII Encuentro Nacional de Experiencias en Enseñanza de la Biología y la Educación Ambiental. III Congreso Nacional de Investigación en Enseñanza de la Biología.

perfiles dominantes y muchos los de baja frecuencia. En ellos predominan los biólogos, licenciados en ciencias naturales, médicos, odontólogos y químicos (ver Tabla 3).

Tabla 3. Perfiles dominantes de maestros de Biología
\begin{tabular}{|l|c|}
\hline FORMACION PROFESIONAL & FRECUENCIA \\
\hline Biología & 91 \\
\hline Cirujano dentista & 80 \\
\hline Ciencias naturales & 47 \\
\hline Medicina & 45 \\
\hline Otra & 17 \\
\hline Veterinario & 16 \\
\hline Técnico & 9 \\
\hline Agrónomo & 5 \\
\hline Ingeniería & 5 \\
\hline Químico & 4 \\
\hline Derecho & 1 \\
\hline Enfermería & 1 \\
\hline Homeopatía & 1 \\
\hline Ciencias de la educación & 1 \\
\hline
\end{tabular}

Una ventaja de la diversidad de perfiles, al menos los relacionados con ciencias de la salud (médicos, odontólogos), es que el currículo tiene un enfoque dual, fisiologista y biologicista (evolutivo). Un biólogo no recibe ambas formaciones como un médico tampoco la tiene. El único perfil dual es el del biólogo o licenciado en ciencias naturales de la normal superior.

\section{Discusión}

En el ámbito mundial, en Europa la existencia de modelos paralelos de formación docente para educación post-primaria se debe a la falta de empleo de profesionistas en su ámbito, por ello su número es bajo y se recurre a ello sólo en asignaturas especializadas. Para ingresar al servicio docente se deben de cursar estudios de pedagogía o tener estudios de posgrado. En Latinoamérica, sin embargo la frecuencia de profesores habilitados de formación consecutiva es más abundante por desempleo, México no es la excepción.

En México, los reportes de investigación son fundamentalmente del DF, puesto que a nivel nacional los datos son pocos, el INEE (2015) reporta datos que corresponden al 2004, la composición de la planta docente de profesores de educación secundaria siempre ha sido mixta, de formación simultánea especializada y consecutiva no específica. El incremento de la población de maestros universitarios dentro de secundarias se da a la par del desempleo. 
Bio - grafia. Escritos sobre la Biología y su Enseñanza. ISSN 2027

Edición Extraordinaria. p.p. 970- 981

Memorias del VIII Encuentro Nacional de Experiencias en Enseñanza de la Biología y la Educación Ambiental. III Congreso Nacional de Investigación en Enseñanza de la Biología.

Por ello la tendencia es a incrementar la diferencia entre universitarios y normalistas -de acuerdo con la Tabla 1, para el caso del Distrito Federal los universitarios están entre el $60 \%$ y $80 \%$, mientras que los normalistas entre el $20 \%$ y $40 \%$-. La falta de especificidad de los profesores de ciencias naturales se agudiza al presentarse el fenómeno del doble arbitrario. Profesionistas no formados para la docencia se les habilita para ello y dentro de las escuelas, se les dispersa su área de desarrollo conceptual, diluyendo la propia. El mismo efecto se da en los profesores especializados de la normal, quienes dan diversas asignaturas.

Por lo que respecta a los docentes de Biología, como se puede observar en la Tabla 3, los perfiles dominantes están relacionados con el área químico biológica, pero dentro de su diversidad se puede encontrar perfiles no sólo fuera del ámbito químico biológico, sino del científico o técnico, como licenciados en derecho, o pedagogos.

\section{Conclusiones}

Se concluye que los perfiles profesionales de los docentes que imparten Biología en escuela secundaria siguen la tendencia de mayor frecuencia de universitarios y menor de normalistas. Sus perfiles son diversos pero se observa dominancia, los dominantes pertenecen al área química biológica y son Biólogos, Licenciados en ciencias naturales, Médicos, Odontólogos y Químicos. En los maestros en servicio conviven los modelos de formación simultánea (normalista) y consecutiva (habilitado). Debido a la doble situación que se sigue como tradición dentro de las escuelas secundarias, se diluye la especificidad de la formación para la enseñanza de los normalistas, ya que atienden cualquier materia de ciencias, al igual que los maestros habilitados. Lo que diluye la especialización del docente y la dispersión de su conocimiento disciplinar, ello coincide con el logro académico reportado para la materia.

La diversidad de perfiles, al menos en los dominantes exceptuando a los químicos, es que tienen la posibilidad de atender una de las prioridades educativas del nivel y de la asignatura, promover el cuidado de la salud. Tantos perfiles diferentes dentro de la 
Bio - grafia. Escritos sobre la Biología y su Enseñanza. ISSN 2027

Edición Extraordinaria. p.p. $970-981$

Memorias del VIII Encuentro Nacional de Experiencias en Enseñanza de la Biología y la Educación Ambiental. III Congreso Nacional de Investigación en Enseñanza de la Biología.

enseñanza de la biología en secundaria, es consecuencia de las condiciones sociales del país, bajo desarrollo y alto desempleo, por lo que el magisterio se vuelve un receptor de profesionistas que de otra forma estarían desempleados. Esa situación social, redunda en el aspecto pedagógico y más aún en el educativo, pues en tanto que un normalista está formado como educador, el universitario no normalista no lo está, presentando desventajas de especificidad disciplinar, falta de formación pedagógica y sin noción de su papel de educador antes que instructor.

\section{Bibliografía}

Arnaut, A. (1998). Historia de una profesión: maestros de educación primaria en México. México: SEP.

Blancas, J. (2010). La Práctica Docente en Ambientes Tecnológicos para la Enseñanza de las Ciencias Experimentales a partir de las Concepciones de los Profesores sobre Ciencia, Aprendizaje y TIC. Tesis de Licenciatura. México: Universidad Pedagógica Nacional.

Esteve, J.M. (2006). La profesión docente en Europa: Perfil, tendencias y problemática. La formación inicial. Revista de Educación -Monográfico sobre la formación inicial del profesorado. Artículo Macro. Ministerio de Educación, Cultura y Deporte Español-. 340(2), 19-86.

Flores-Camacho, F., Gallegos-Cazares, L., García-Franco, A., Vega-Murguía, E. y GarcíaRivera, B. (2007). El conocimiento de los profesores de ciencias naturales de secundaria: un estudio en tres niveles. Revista Iberoamericana de Educación, 43 (3), 1-19.

INEE. (2010). México en PISA 2009. 19. Edición. México: Instituto Nacional para la evaluación Educativa.

INEE. (2015). Los docentes en México. Informe 2015.19 edición. México: Instituto Nacional para la Evaluación de la Educación.

Martínez, L. (1997). Un acercamiento a la evaluación comparativa del docente de Biología en secundaria. Tesis de Licenciatura. México: Universidad Pedagógica Nacional. 
Bio - grafia. Escritos sobre la Biología y su Enseñanza. ISSN 2027

Edición Extraordinaria. p.p. $970-981$

Memorias del VIII Encuentro Nacional de Experiencias en Enseñanza de la Biología y la Educación Ambiental. III Congreso Nacional de Investigación en Enseñanza de la Biología.

Martínez, L., Más, L. y Paz, V. (2014). ¿Quiénes enseñan biología en las escuelas secundarias generales de México? Un caso, Iztapalapa, ciudad de México. Ponencia. VI Congreso Internacional sobre Formación de Profesores de Ciencias. Bogotá, Colombia.

Murillo, J. (2006). Modelos innovadores para la formación inicial docente. Una propuesta por el cambio. Santiago de Chile: ORELAC/UNESCO

OEI. (2003). Organización y estructura de la formación docente en Iberoamérica. Documento de trabajo. España: Organización de Estados Iberoamericanos. Para la

Educación, la Ciencia y la Cultura.

Quiroz, R. (2002). Currículum y prácticas de enseñanza en la escuela secundaria. En $G$. Ynclán, Todo por hacer. Algunos problemas de la Escuela Secundaria (69-92). México: Centro de Investigación para el Éxito y la Calidad Educativa, S.C.

Rico, C. (2006). Las Concepciones Alternativas de los Profesores de Educación Secundaria sobre la Evolución en los Seres Vivos. (Tesis de Maestría). México: Universidad Nacional Autónoma de México.

Rodríguez-Pineda, D.P. (2007). Relación entre concepciones epistemologias y de aprendizaje, con la practica docente de los profesores de ciencias, a partir de las ideas previas en el ámbito de la física. (Tesis de Doctorado). México: Universidad Pedagógica Nacional.

Sandoval, E. (2001). Ser profesor de secundaria en México: condiciones de trabajo y reformas educativas. Revista Iberoamericana de Educación, 25, 83-102. Recuperado de http://www.rieoei.org/rie25f.htm

SEP. (2012) Profesiograma. México: SEP - SNTE.

Tarango, E. (2012). Los docentes y la enseñanza de la historia con los programas de la reforma a la educación secundaria 2006, reproducción o resistencia. (Tesis de Doctorado. México: Universidad Pedagógica Nacional. 